\title{
Prevention and Control of Cardiovascular Diseases by Beneficial Use of Islamic Lifestyle
}

\author{
Mohammad Rabbani Khorasgani* \\ University of Isfahan, Iran
}

*Corresponding author: Mohammad Rabbani Khorasgani, Department of Biology, University of Isfahan, Iran.

Received Date: February 18, 2019

Published Date: March 07, 2019

\section{Introduction}

In recent decades, the advancement of human researches and public health activities resulted to infectious diseases control, therefore, the morbidity and mortality rates of many important infectious diseases in the world declined. Although, the risk of development and spreading of emerging, re- emerging, and drugresistantinfectious diseases are important, buthuman industrialized life and life style changes have resulted in new challenges for human health specially in the field of non- communicable diseases (NCDs): cardiovascular diseases (CVDs), cancers, obesity and etc. Non-communicable diseases, are the major causes of death in the world, representing $63 \%$ of all annual deaths (more than 36 million people). It is estimated that 17.9 million people died from CVDs in 2016, representing $31 \%$ of all global deaths [1]. On the other hand, disease development is influenced by different host, environment and microorganisms related factors, therefore, the pay attention to predisposing cultural, social, economic and religious factors is necessary for human health promotion as a multidisciplinary subject.

\section{What is Lifestyle?}

Lifestyle is a way or style used by people in their life in special time and place and includes behaviors and functions of individuals. Lifestyle is formed in specific geographical, economic, political, cultural and religious context and influenced by them $[2,3]$.

\section{The Influence of Lifestyle on Different Aspects of} Human Life

Lifestyle is an important and very effective agent in human beings life including the human-human, and human-environment interactions. If human regarded as whole, the thoughts, perspectives and practices affect his body and soul directly or non-directly. Many philosophical, religious documents and excellent scientific findings in psychology, biology and medicine favor it [4]. Therefore, human health is influenced by lifestyle, also. WHO has estimated that $60 \%$ of related factors to individual health and quality of life are correlated to lifestyle [3]. Pay attention to complex, but, define networks in neuro immune endocrine communication could be elucidate some aspects of lifestyle effects on human health. Lifestyle management or promotion may be regarded as effective action for health promotion.

\section{Influences of Lifestyle on Cardiovascular Diseases}

Lifestyle may be influence predisposition, development, exacerbation or prevention and control of cardiovascular diseases. Most cardiovascular diseases can be addressed by lifestyle-related risk factors such as unhealthy diet and obesity, physical inactivity, tobacco use, and harmful use of alcohol [1]. On the other hand, some lifestyle-related factors may be beneficially effective in prevention and control of CVDs. Diet management, physical activity and good pattern of sleepiness and awaking are some samples of these factors.

\section{Islamic Lifestyle and Cardiovascular Diseases}

Islamic lifestyle affecting different aspects of human body and soul, can influence CVD development, prevention and control. In this article, some important characteristics of Islamic lifestyle with potential effect on CVDs are presented as the following items:

\section{Diet and nutrition style}

Diet has been regarded as one of the greatest factors in lifestyle and has a direct relation with health. Imbalanced diet, overeating and their consequences like obesity are the common healthy problem with negative effect on heart health. Unhealthy nutrition style can be measured by some indexes such as Body Mass Index (BMI) that could be related to nutrition and metabolism of human body [3]. Many studies indicate that dietary intake affects CVDs development and progression [5]. On the other hand, it is believed that cultural and religious factors affect different aspects of nutrition such as diet quantity and quality, food sources and etc. 
In Quran, the holy book of Muslims, and some other Islamic texts have mentioned some guide lines, important points and examples related to spiritual and physical aspects of human health especially about human nutrition that may be regarded as Islamic nutrition style. Some important findings about human nutrition concluded from Islamic texts are presented as the following:
A. The nutrition important and its effect on human life
B. Essential guidelines for foodstuffs exploitation:

I. Exploitation of different sources (herbal and animal) for nutrition

II. "Halal" or permissible foods such as vegetables, fruits, meat of lamb, goat, beef, chicken and fish

III. "Haram" or forbidden foods such as alcoholic drinks, pork, carcasses meat, blood and filthy materials

IV. Necessity of fasting during Ramadan

V. Necessity of carefulness about foodstuff usage and evaluation of the benefits and harms of potential foods

VI. Forbidden of overeating and food extravagance

VII. The emphasis on limitation of meat consumption to the amount of human requirement

VIII. -Emphasis on beneficial and curative effect of honey for human health

It is suggested that Islamic lifestyle in nutrition, expresses beneficial effects for normal nutrition and prevention of obesity and nutritional disorders and therefore heart health [4]. Some traditional foods as recommended foods in Islam may be essential role in people health and longevity. Mediterranean diet and natural foods containing probiotics (such as dairy products) and prebiotics (such as honey) with health beneficial effects involve in prevention of CVD. [6]. Probiotics and prebiotics can ameliorate CVD through improvement of gut microbiota, which in turn leads to cholesterollowering effects [7]. Honey with antioxidant, anti-inflammatory, and antimicrobial activities expresses beneficial effects on human health. More studies are exploring other potential activities of honey such as its effect on blood sugar, body weight, lipid profile, C-reactive protein, nitric oxide, pro inflammatory prostaglandins, and homocysteine. Growing scientific evidences support the use of honey in patients with diabetes, HTN, dyslipidemia, obesity, and CVD and clinical and preclinical studies on potential influence of honey on cardiovascular risk factors, although more clinical and controlled studies are needed for final conclusion [8]. On the other hand, based on studies across mice and humans, it is suggested that the modern Western lifestyle common aspects, including high fat diets, can persistently alter commensal microbial communities. Those microbial disturbances may increase obesity as one of CVD risk factors [9].

\section{The religious beliefs and practices}

Cultural and religious beliefs, and regulations may be regarded as some important factors affecting human lifestyle, therefore, pay attention to relationships between above factors, lifestyle and
CVD development, progress, prevention, control and treatment is necessary. Different religious practices including some private and / or communications and multidisciplinary works express important effects on development or control, prevention and treatment of CVD. Understanding religious practices may allow public health officials to communicate with populations, more effectively target them and encourage CVD prevention using religious beliefs and so on. In Islam, there are some essential rules and recommendations with controlling effect against CVD as the following:

I. Emphasis on the necessity of pay attention to keep healthy and preventing harmful health effects [4]

II. Emphasis on human ethical improvement with trust and reliance on Allah, tolerance, hopefulness for the future and necessity of pay attention to the rights of others that may be resulted to stress management [10]

III. The emphasis on spiritual health strengthening, piety and abstinence "Taghva"

IV. Encouraging the marriage and regarding the family lifestyle as important principles for management of essential unit of community

V. Encouraging the acquisition of health-related sciences and underlining that: "for any pain, there is a cure"

VI. The emphasis on the importance of medicine and preventing the non-scientific intervention on human health

VII. Insistence on: "prevention is better than cure" [4,11,15]

VIII. The beneficial effects of some religious practices such as praying especially official praying "Salat" on CVD control, prevention and pain relief [12]

IX. Prohibition of Jobs with potential harmful effects [11]

\section{Marital status and family formation}

Marital status and family formation style may influence CVD development and progress. There are some reports about the relation between marital status and risk of cardiovascular diseases. Based on systematic review and meta-analysis of 34 studies with more than two million participants by Wong et al (2018), being unmarried (never married, divorced or widowed) was associated with increased odds of CVD, coronary heart disease (CHD), CHD death, and stroke death compared with married participants. Single men and women with myocardial infarction had increased mortality compared with married participants [13]. Sexual relationship, marital status and family formation style may be influenced by many factors such as religion, culture, custom, social media, regulations, economy and etc. Religion may be regarded as an important factor affecting human lifestyle in these fields. Abrahamic religions especially Islam, express essential phrases for sexual relationship, marital status and family formation style. Some important findings from Islamic lifestyle with potential effect on these fields are included:

-Encouraging the marriage at the first suitable time : After family formation, human relationships would be developed at higher scale and different physiologic and spiritual needs may 
be managed correctly. Family formation could prepare best situation for safe sex as it recommended by Abrahamic religions (Christianity, Judaism and Islam) and some others to saving sex for marriage. Health reasons, moral beliefs and to get a happier and more romantic marriage, support of it [14]. A healthy, happy and qualified lifestyle in the family including good nutrition, physical activity, love, spirituality support and respect each other would be helpful for improvement of individual and society health and can decrease risk factors of CVDs. Some important rules mentioned in Islamic texts about sexual relations with potentially beneficial effects on human health especially CVD prevention may be presented as the following:

a. Not unrestrained sexual activity, nor sexual asceticism but legal sexual activities that restricted to marriage

b. Prohibition of unlawful and illicit relationships (adultery, pederasty and same-sex relationships)

c. Encouraging the chastity and forbidden of indecent behaviors

d. Prohibition of alcoholic beverages and therefore prevention of uncontrolled behaviors

e. Punishment for overt unlawful sexual relations and sex crimes $[11,15]$.

It is seemed that Islamic lifestyle in the field of sex relations, marriage and family formation express beneficial effects on CVD prevention.

\section{Sleep and awakening pattern}

Sleep and awakening pattern is an important part of the lifestyle. Although the effects of sleep deprivation on human organs have been obscure, recent epidemiological studies have revealed relationships between sleep deprivation and hypertension (HT) and coronary heart disease (CHD) [16]. In Islamic lifestyle, the properly time management between working, sleeping, spiritual duties and recreation and entertainment activities is recommended.

\section{Social lifestyle}

Interpersonal relationships and people communications within their immediate surroundings or general public are known as social life [32]. But some researchers limit social life as the part of a person's time spent doing enjoyable things with others [17]. In this article, the general concept of social life has regarded excluding family which discussed separately.

Social issues constitute an important and often overlooked component of factors associated with the etiology, incidence, and evolution of CVD [18]. Everybody life is included personal and social aspects with different quantitative and qualitative degrees. It is believed that human as a social being needs to communicate with other people. Therefore, suitable social life induces beneficial effects on human health and social isolation and loneliness may negatively affect human health aspects. Social isolation is an objective measure of the lack of social connections or interactions, but, loneliness, as the disagreement between a person's desired and actual social relationships, is an emotional response to social isolation. Consequently, loneliness is thought to be more related to relationship quality than quantity. Cultural, social and demographic changes have led to an increased prevalence of loneliness and social isolation in many societies specially modern societies. With increasing lifespan, the number of people aged 60 years and older has tripled since 1950 . Older age is usually associated with reduced social interactions, longer periods of time living alone, and higher prevalence of loneliness. However, loneliness can be experienced at all stages of life. The prevalence of loneliness has increased with delayed marriage, increased two-income households, and increased residence in single-family homes. In addition, the Internet has completely changed the way people live and interact. Despite increased digital connectivity, more people are experiencing social isolation. Social isolation and loneliness are clearly risk factors for cardiovascular disease (CVD) such as elevated blood pressure and atherosclerosis. Strong social relationships can increase the likelihood of survival by as much as $50 \%$ relative to individuals whose relationships are weaker. Perceived social isolation (PSI) has damaging effects on human health and inducing behavioral alteration, including physical inactivity, smoking, and sleep disruption, increased depressive behavior, chronic stress and etc. [19]. In Islamic lifestyle, there are essential preparations to prevent people loneliness such as: Emphasis on marriage, religious and social duties: the care and support of parents and elderly people, visiting the sick, collective worship practices such as congregational prayer" Salat", therefore, Islamic social lifestyle helps CVD prevention and control by reducing their risk factors.

\section{The control of sedentary behavior and recreation activities}

The majority of prospective studies of sitting time has shown that greater sedentary time is associated with an increased risk of fatal and non-fatal CVD [20]. On the other hand, the type of leisure spending by different activities, recreation and entertainments as an important part of human life, depends on the people lifestyle. Outdoor recreation: camping, playing sports and mountain climbing, travel and tourism events with different purposes: religious, economic, entertainment increase human mobility and limit sedentary [21]. In Islamic lifestyle suitable recreation and entertainments activities are recommended especially if they would be joined with thinking, people communication, helping together, friendship with the nature and conservation of creature's rights.

\section{Reducing high-risk behaviors: alcohol consumption, smoking, drug abuse \& addiction}

Alcohol consumption: Alcohol misuse has been part of many human societies for centuries. In 2016, alcohol use was the seventh leading risk factor for both deaths and disability-adjusted life-years (DALYs) [22]. Between 2006 and 2010 in the United States, excessive alcohol consumption resulted in approximately 88,000 deaths (data based on 11 U.S. States) with death rate: 28.5 per 100,000 population. The potential life lost attributed to alcohol averaged about 2.5 million years annually. In addition, the estimated cost of excessive drinking including: loss of productivity, health care costs and criminal justice was $\$ 223.5$ billion in 2006. Many evidences 
indicate that alcohol has a systemic effect on different organs of body. Patients with a history of heavy acute or chronic alcohol use, have higher rates of hospitalizations, longer hospital stays, major complications of poly trauma and bleeding disorders, increased mortality, higher intensive care unit admissions, and greater postoperative complications, compared with patients with no history of alcohol use [23]. Harmful use of alcohol is an important risk factor for CVD development [1].

Drinking too much alcohol can raise the levels of some fats in the blood (triglycerides). It can also lead to high blood pressure, heart failure and an increased calorie intake can lead to obesity. Excessive drinking and binge drinking can lead to stroke and other serious problems include fetal alcohol syndrome, cardiomyopathy, cardiac arrhythmia and sudden cardiac death [24]. The significant relationship between high frequency of alcohol drinking and high frequency of binge drinking with high systolic and diastolic blood pressure (SBP \&DBP) are reported [25]. Some researchers have defined risk thresholds for alcohol consumption, but for cardiovascular diseases, there were no clear risk thresholds below which lower alcohol consumption stopped being associated with lower disease risk [26]. Prohibition of eating potentially deleterious materials known as "Haram" substances such as alcohol in Islam may decline the rate of CVD [4].

Smoking: Smoking as a habit may be an inalienable part of life of some peoples. Depending on the quantity and quality of smokedmaterial and the consumer situation, it will be induce a range of harmful effects against human health. Tobacco is the most common substance smoked especially with cigarettes. Cigarette smoking is an important risk factor for CVD [27]. After clearing the tobaccorelated health losses, many jurisprudents (Experts in Islamic law) have regarded them as deleterious "Haram" substances [29].

Drug abuse \& addiction: Substance abuse still remains one of the major problems in the world with important social, economic and health consequences and millions of people abusing legal and illegal drugs today. Substance abuse is one of the major problems in the world today with an estimated 200 million people abusing illegal drugs regularly. In the USA, according to the 2005 National Survey on Drug Use and Health, about 110 million Americans age 12 years and older (46\%) used at least one illicit drug (e.g., amphetamines, cocaine, heroin, or marijuana) in their lifetime. Substance abuse imposes enormous sociopolitical, economic, and health costs to the society. Legal and illegal substance abuse costs the American society an estimated one-half a trillion dollars annually [14]. Both illicit and prescription drugs present many risks to individuals who abuse them. Side effects from abusing drugs can range from mild to deadly, and they will differ depending on the type of substance and the method of taking it. Drug abuse affects the brain that may lead to erratic, dangerous behaviors, social and emotional problems, and the development of addiction. Drug abuse in its many forms can profoundly impact the body-damaging a number of organ systems and influencing a decline in general physical health. The cardiovascular system is one system particularly affected by certain drugs of abuse [28].
In Islamic lifestyle, abusing any substance with harmful effects is forbidden [29], therefore, it could limit this risk factor of CVDs.

\section{Conclusion}

What has been above mentioned is indicating essential role of lifestyle on development, transmission, frequency and incidence of CVD. Therefore, carrying out in-depth studies about lifestyle especially Islamic lifestyle and CVD relationship with understanding, thinking deeply and beneficial using of cultural and religious teachings and human experiences will be helpful to take proper decisions for effective modifications in lifestyle, in the process of acquiring healthy communities. It is recommended to study Islamic approach to health carefully for beneficial uses of them for prevention and control of diseases especially CVDs.

\section{Acknowledgement}

None.

\section{Conflict of Interest}

No conflict of interest.

\section{References}

1. https://www.who.int/cardiovascular_diseases/en/

2. Burton R, Sheron N (2018) No level of alcohol consumption improves health by use of methodological enhancements of previous iterations. 392: 987-988.

3. Lawrence A David, Arne C Materna, Jonathan Friedman, Maria I Campos Baptista, Matthew C Blackburn (2014) Host lifestyle affects human microbiota on daily timescales. Genome Biology 15: R8.

4. Nam S, Whittemore R, Jeon S, Davey Rothwell MA, Latkin C (2015) High blood pressure and related factors among individuals at high risk for HIV/sexually transmitted infections. Journal of Clinical Hypertension 18(6): 572-80.

5. Biazar Shirazi A (1994) Medical- Islamic jurisprudence new treatise, Spiritual medicine, Daftar Nashr Farhang Eslami, Tehan, Iran.

6. Rabbani M (2005) Culture and Health: Science and Religiousness. Basij Asatid Organization Publications.

7. Yoo J Y, Kim SS (2016) Probiotics and Prebiotics: Present Status and Future Perspectives on Metabolic Disorders. Nutrients 8(3): 173.

8. Al Waili N, Salom K, Al Ghamdi A, Ansari MJ, Al Waili A (2013) Honey and cardiovascular risk factors, in normal individuals and in patients with diabetes mellitus or dyslipidemia. Med Food 16(12): 1063-1078.

9. Asghar Dalvandi, Sadat Seyed Baghr Maddah, HamidReza Khankeh, Shahriar parvaneh, Fazel Bahrami (2013) The Health-Oriented Lifestyle in Islamic Culture. J Qual Res Health Sci 1(4): 332-343.

10. Biazar Shirazi A (1995) Medical-Islamic jurisprudence new treatise. Treatment related subjects Daftar Nashr Farhang Eslami Tehan Iran.

11. Rabbani Khorasgani M, Shafiei R (2017) Traditional yogurt as a source of lactobacilli and other lactic acid bacteria in Iran. Shah NP editor, Yogurt in health and disease prevention, pp. 285-94.

12. Martínez García M, Salinas Ortega M, Estrada Arriaga I, Hernández Lemus E, García Herrera R (2018) A systematic approach to analyze the social determinants of cardiovascular disease. PLoS ONE 13(1): e0190960.

13. Amy Wesolowski, Gillian Stresman, Nathan Eagle, Jennifer Stevenson, Chrispin Owaga (2014) Quantifying travel behavior for infectious disease research: a comparison of data from surveys and mobile phones. Scientific Reports 4: 5678. 
14. Ford ES, Carl J Caspersen CJ (2012) Sedentary behavior and cardiovascular disease: a review of prospective studies. International Journal of Epidemiology 41(5): 1338-1353.

15. Rabbani Khorasgani M (2018) Effectiveness of the lifestyle modifications in prevention and control of sexually transmitted diseases (STDs): Focus on Islamic lifestyle. Clin J Obstet Gynecol 1: 056-057.

16. Moeini M (2014) Patients' religious beliefs in cardiac pain situations: A qualitative research. J Qual Res Health Sci 3(1): 104-13.

17. https://www.merriam-webster.com/dictionary/social\%20life

18. Khalsa JH, Treisman G, Mc Cance Katz E, Tedaldi E (2008) Medical consequences of drug abuse and co-occurring infections: research the National Institute on Drug Abuse. Substance abuse 29(3): 5-16.

19. Xia N, Li H (2018) Loneliness, social isolation, and cardiovascular health. Antioxid Redox Signal 28: 837-851.

20. Farhud DD (2015) Impact of lifestyle on health. Iranian Journal of Public Health 44(11): 1442-1444.

21. Trevejo Nunez G, Kolls JK, De Wit M (2015) Alcohol use as a risk factor in infections and healing: A clinician's perspective. Alcohol Research: Current Reviews 37(2).

22. Bowen KJ, Sullivan VK, Kris-Etherton PM, Petersen KS (2018) Nutrition and Cardiovascular Disease-an Update. Current Atherosclerosis Reports 20(2): 8.
23. Stahre M, Roeber J, Kanny D, Brewer RD, Zhang X (2014) Contribution of excessive alcohol consumption to deaths and years of potential life lost in the United States. Prev Chronic Dis 11:E109.

24. https://www.heart.org/en/healthy-living/healthy-eating/eat-smart/ nutrition-basics/alcohol-and-heart-health

25. Nagai M, Hoshide S, Kario K (2010) Sleep duration as a risk factor for cardiovascular disease- a review of the recent literature. Current Cardiology Reviews 6(1): 54-61.

26. Wong CW, Kwok CS, Narain A, Gulati M, Mihalidou AS (2018) Marital status and risk of cardiovascular diseases: a systematic review and meta-analysis. Heart 104: 1937-1948.

27. https://www.heart.org/.../healthy-lifestyle/quit-smoking-tobacco

28. https://drugabuse.com/drug-addiction-health-issues/cardiovascular/

29. Wood AM, Kaptoge S, Butterworth AS, Willeit P, Warnakula S (2018) Risk thresholds for alcohol consumption: combined analysis of individualparticipant data for 599912 current drinkers in 83 prospective studies. Lancet 391: 1513-1523.

30. Jimmy IM (2017) The Arguments for and against saving sex for marriage.

31. Rabbani Khorasgani M, Atyabi SR (2017) Renal diseases and Islamic religious beliefs and practices. Austin Journal of Nephrology and Hypertension 5: 076.

32.https://en.wikipedia.org/wiki/Social_life 